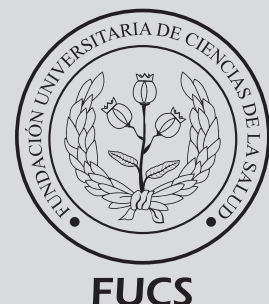

\title{
Estudio de los resultados del contagio por COVID-19 a nivel mundial
}

\section{Analysis of the results of the transmission of COVID-19 worldwide}

a Ingeniero. Magister en Gestión de la Tecnología Educativa, Especialista en Administración de la Informática Educativa.

Docente de matemáticas e Investigador, Secretaría de Educación de Soacha, Cundinamarca.

\section{R ES U M EN}

Introducción: al cumplirse seis meses del primer contagio por coronavirus COVID-19 en el mundo, se realizó un estudio de investigación de los resultados de transmisión de la enfermedad por su distribución a nivel mundial. Objetivo: comparar si hay diferencias en los promedios de personas con contagio por COVID-19 entre los diferentes países del mundo. Materiales y métodos: se utilizó la base de datos de las personas infectadas por el COVID-19 con la información acumulada entre el 28 y 30 de junio de 2020. Se realizó a partir del análisis estadístico de los datos aplicando la ANOVA, y una prueba post hoc de Duncan, se utilizó el paquete estadístico SPSS v.25. Resultados: a partir del análisis estadístico de los datos aplicando la comparación de medias con el ANOVA, el p-valor de las pruebas, para la variable contagios es de 0,000, este es menor a $a=0.05$, de esta manera se rechaza Ho, es decir que para al menos uno de los promedios es diferente de los demás del resultado obtenido para los diferentes países del mundo por contagio de personas por COVID-19. Conclusiones: la mitad de los contagios del mundo se han registrado en Estados Unidos y Europa, pero está creciendo rápidamente en toda América. En América Latina el ritmo de nuevas infecciones se ha acelerado desde mediados de mayo, y está aumentando especialmente en Brasil, México y Chile, según la Organización Mundial de la Salud.

Palabras clave: COVID-19, coronavirus, transmisión de enfermedad infecciosa; pandemia.

(C) 2020 Fundación Universitaria de Ciencias de la Salud - FUCS. Este es un artículo Open Access bajo la licencia CC BY-NC-ND (http://creativecommons.org/licenses/by-nc-nd/4.0/).

\section{INFORMACIÓN DEL ARTÍCULO}

Historia del artículo:

Fecha recibido: julio 7 de 2020

Fecha aceptado: julio 10 de 2020

\footnotetext{
Autor para correspondencia.

Jorge Enrique Díaz Pinzón jediazp@unal.edu.co
}

DOI

10.31260/RepertMedCir.01217372.1089 


\section{A BSTRACT}

Introduction: after six months of detecting the first case of Coronavirus disease 2019 (COVID-19) in the world, a research study on the transmission of the disease by its global distribution, was carried out. Objective: to determine if there are differences in the averages of people infected with COVID 19 between countries around the world. Materials and Methods: the database including people infected with COVID-19 was used, with the information collected between June 28 and 302020. Data collected by applying the ANOVA and a Duncan post hoc multiple comparisons test was used for statistical analysis, in the SPSS v.25 statistical package. Results: from the statistical analysis of data applying the ANOVA mean comparison, it was determined that the $p$ value for the variable, transmission, is equal to 0.000 , which is less than $a=0.05$, thus, the hypothesis (Ho) is rejected, which means that at least for one of the averages it is different from the rest of the results obtained for infected people with COVID-19 from nation to nation. Conclusions: half of the global number of cases have been registered in the United States and Europe, but are rapidly increasing across America. In Latin America the rate of new cases has accelerated since mid-May, and is rising predominantly in Brazil, Mexico and Chile, according to the World Health Organization.

Key words: COVID-19, coronavirus, infectious disease transmission; pandemic.

(C) 2020 Fundación Universitaria de Ciencias de la Salud - FUCS. This is an open access article under the CC BY-NC-ND license (http://creativecommons.org/licenses/by-nc-nd/4.0/).

\section{INTRODUCCIÓN}

Si bien la pandemia a causa del nuevo coronavirus a nivel mundial se encuentra en una fase de crecimiento todavía y en el momento a 30 de junio de 2020 se contabilizan oficialmente 68.652 casos, 2.237 muertes, 27.360 recuperados y 30.055 infecciones activas, estas cifras, en promedio, resisten comparaciones con lo que ocurre en el mundo.

El nuevo coronavirus 2019 es un virus de ARN de sentido positivo no fraccionado que corresponde a la familia Coronaviridae-Nidovirales. ${ }^{1}$

Desde la aparición de la enfermedad por coronavirus 2019 (COVID-19) en diciembre 2019, la enfermedad se ha transformado ágilmente en una pandemia que intimida a las sociedades de todo el mundo. ${ }^{2}$

La pandemia de la enfermedad por coronavirus 2019 (COVID-19) ha sido un auténtico reto para los sistemas de salud y los médicos. ${ }^{3}$

La Organización Mundial de la Salud ha señalado la rápida propagación de COVID-19 en todo el mundo como una emergencia de salud pública mundial. Es bien sabido que la propagación de la enfermedad está influenciada por la gente, la voluntad de adoptar conductas preventivas de salud pública que a menudo se asocian con la percepción pública del riesgo. ${ }^{4}$

Sin embargo, nuestros descubrimientos, que presentan la primera evidencia semejante de cómo las personas descubren el riesgo de COVID-19 en todo el mundo, enseñan claramente que las percepciones se correlacionan insistentemente con una serie de factores experimentales y socioculturales en todos los países. ${ }^{4}$

A medida que los países están lidiando la pandemia de COVID-19, una cosa se está esclareciendo, el mundo posterior al coronavirus será diferente en muchos semblantes desde el punto de vista económico, social y de salud. Nadie saldrá de esta crisis sin perder algo.

Las intimidaciones anteriores a las libertades individuales y sociales no podían trocar nuestra forma de vida, pero ahora este importante activo de nuestra civilización está gravemente amenazado. ${ }^{5}$

De acuerdo con Díaz en un estudio de investigación en Colombia, determinó que se presentó mayor contagio de personas por COVID-19 con la apertura global y sistemática de varios sectores de la economía que cuando se estuvo en cuarentena obligatoria. Primero, sugerimos que las prácticas nacionales en su batalla contra COVID-19 dependen del camino y del contexto.

No existe una solución única para todos. No obstante, algunos ejercicios comunes de distanciamiento social y encierro ciertamente pueden aplanar la curva del aumento de los casos confirmados y las tasas de mortalidad, son las especificidades locales de muchos otros tipos, como las estructuras económicas, los paradigmas de gobernanza y las preferencias culturales, las que prescriben el éxito de la enfermedad lidiando contra los esfuerzos. ${ }^{7}$

\section{METODOLOGÍA}

El trabajo de investigación se realizó mediante un enfoque cuantitativo según Hernández ${ }^{8}$, es un proceso secuencial y probatorio; se miden las variables en un establecido contexto; se analizan las mediciones alcanzadas (con frecuencia empleándose métodos estadísticos) y se instaura una serie de conclusiones con respecto a las hipótesis. 
De acuerdo con Shuttleworth, citado por Díaz los experimentos cuantitativos utilizan un formato estándar con algunas pequeñas diferencias interdisciplinarias para generar una hipótesis que será probada o desmentida. Esta hipótesis debe ser demostrable por medios matemáticos y estadísticos y constituye la base alrededor de la cual se diseña todo el experimento.

\section{P OB L ACIÓN}

Esta investigación se centró en los 40 países del mundo con mayor nivel de contagio, con la información proveniente de Google ${ }^{10}$ en el periodo comprendido entre el 28 y 30 de junio 2020 (tabla 1).

Tabla 1. Media de contagios, fallecidos y casos por 1 millón de personas a nivel mundial

\begin{tabular}{|c|c|c|c|c|}
\hline Posición & País & Contagio & Fallecidos & Casos por 1 millón de personas \\
\hline 1 & Estados Unidos & 2668647 & 8098 & 128581 \\
\hline 2 & Brasil & 1370488 & 6485 & 58385 \\
\hline 3 & Rusia & 647849 & 4415 & 9320 \\
\hline 4 & India & 566840 & 417 & 16893 \\
\hline 5 & Reino Unido & 312654 & 4706 & 43730 \\
\hline 6 & Perú & 282365 & 8788 & 9504 \\
\hline 7 & Chile & 279393 & 14622 & 5688 \\
\hline 8 & España & 249271 & 5292 & 28355 \\
\hline 9 & Italia & 240578 & 3993 & 34767 \\
\hline 10 & Irán & 227662 & 2732 & 10817 \\
\hline 11 & México & 220657 & 1743 & 27121 \\
\hline 12 & Pakistán & 209337 & 955 & 4303 \\
\hline 13 & Turquía & 199906 & 2404 & 5131 \\
\hline 14 & Alemania & 195747 & 2354 & 9029 \\
\hline 15 & Arabia Saudita & 190823 & 5577 & 1649 \\
\hline 16 & Francia & 164801 & 2457 & 29843 \\
\hline 17 & Sudáfrica & 151209 & 2573 & 2657 \\
\hline 18 & Bangladesh & 145483 & 864 & 1847 \\
\hline 19 & Canadá & 104151 & 2742 & 8591 \\
\hline 20 & Colombia & 97846 & 1981 & 3334 \\
\hline 21 & Qatar & 96088 & 34976 & 113 \\
\hline 22 & China & 83531 & 60 & 4634 \\
\hline 23 & Suecia & 68451 & 6624 & 5333 \\
\hline 24 & Egipto & 66754 & 666 & 2872 \\
\hline 25 & Argentina & 62255 & 1385 & 1280 \\
\hline 26 & Bielorusia & 62118 & 6599 & 392 \\
\hline 27 & Bélgica & 61361 & 5324 & 9732 \\
\hline 28 & Indonesia & 56385 & 211 & 2876 \\
\hline 29 & Ecuador & 55385 & 3189 & 4502 \\
\hline 30 & Holanda & 50223 & 2878 & 6107 \\
\hline 31 & Irak & 49109 & 1255 & 1943 \\
\hline 32 & Emiratos Arabes & 48246 & 4878 & 314 \\
\hline 33 & Kuwait & 46195 & 7698 & 354 \\
\hline 34 & Singapur & 43907 & 7698 & 26 \\
\hline 35 & Ucrania & 43628 & 1042 & 1147 \\
\hline 36 & Portugal & 41912 & 4078 & 1568 \\
\hline 37 & Oman & 40070 & 8590 & 176 \\
\hline 38 & Filipinas & 36438 & 336 & 1255 \\
\hline 39 & Polonia & 34144 & 890 & 1444 \\
\hline 40 & Suiza & 31652 & 3686 & 1682 \\
\hline
\end{tabular}

Fuente: Google ${ }^{10}$ 


\section{ANÁLISIS ESTADÍSTICO}

Se plantearon hipótesis para la utilización de una prueba de comparación de medias utilizando el ANOVA para la variable contagio por COVID-19, diseñándose como una relación causal y se expone de la siguiente forma:

Hipótesis con el ANOVA

La hipótesis del presente trabajo de investigación se diseña como una relación causal y se enuncia de la siguiente forma:

Hipótesis Alterna (Ha): hay diferencias en el promedio de los puntajes obtenidos por el contagio del COVID-19 entre los diferentes países del mundo.

Hipótesis Nula (Ho): no hay diferencias en el promedio de los puntajes obtenidos por el contagio del COVID-19 entre los diferentes países del mundo.

Prueba Estadística: el valor de significancia de la prueba es de $a=0.05(5 \%)$, si es mayor se acepta la hipótesis nula si es menor se rechaza la hipótesis nula. Comparación entre medias independientes, se utilizó el Software SPSS v 25.0.

Tabla 2. ANOVA- Contagios

\begin{tabular}{|l|l|l|l|l|l|}
\hline & Suma de cuadrados & gl & Media cuadrática & \multicolumn{1}{|c|}{ F } & Significancia \\
\hline Entre grupos & 23955814284212,574 & 39 & 614251648313,143 & 10720,365 & 0,000 \\
\hline Dentro de grupos & 4583811531,333 & 80 & 57297644,142 & & \\
\hline Total & 23960398095743,906 & 119 & & & \\
\hline
\end{tabular}

Fuente: el autor.

\section{RES ULTADOS}

En la (tabla 2), se observa que el p-valor de la prueba es 0,000 es menor a $a=0.05$, de esta manera se rechaza Ho, es decir que para al menos uno de los promedios es diferente de los demás.

Ahora cuando Ho se rechaza en la prueba ANOVA, entonces se procede a realizar una prueba post hoc, en este caso la prueba de Duncan que es un test de comparaciones múltiples, admite comparar las medias de los t niveles de un factor, posteriormente de haber rechazado la hipótesis nula de igualdad de medias mediante la técnica ANOVA $^{11}$ para muestras independientes y observar si hay diferencias entre cada uno de los niveles.

Se puede apreciar en las (tablas 3 y 4), 22 subconjuntos, en ellas se observan en el subconjunto 1 a los países con menor promedio de personas contagiadas por el COVID-19 así sucesivamente hasta llegar hasta el subconjunto 22, en los países con mayor promedio de contagio.

Podemos apreciar que los países con menor promedio de personas contagiadas son: Suiza 31.628, Polonia 33.986, Filipinas 36.110, Omán 39.093, Portugal 41.582, Ucrania 43.197, Singapur 43.675, Kuwait 45.553.

Los países con mayor promedio de personas contagiadas por el COVID-19 son: Estados Unidos con 2'628.930, seguida de Brasil con 1'353.295, Rusia 641.147, India con 584.005, y Reino Unido con 311.923.

A 30 de junio 2020 el número de casos confirmados de coronavirus en todo el mundo fue de 1'0273.001, el de personas recuperadas 5'292.383 y el de muertes 505.295, según el Instituto Nacional de Salud. ${ }^{12}$

La mitad de los contagios del mundo se han registrado en Estados Unidos y Europa, pero el número de contagios está creciendo rápidamente en toda América.

En América Latina el ritmo de nuevas infecciones se ha acelerado desde mediados de mayo, y está aumentando especialmente en Brasil, México y Chile, según la Organización Mundial de la Salud. ${ }^{13}$

En la (figura 1) se aprecia los países con el mayor número de casos por 1 millón de personas a 30 de junio 2020, Chile tiene el mayor índice con 14.622, Perú con 8.788, Estados Unidos con 8.098, Brasil con 6.485, Arabia Saudita con 5.577, y España con 5.292.

En la (figura 2) se observan los países con el mayor número de personas fallecidas a 30 de junio de 2020; lidera los Estados Unidos con 128.581, Brasil con 58.385, Rusia con 47.730, Italia con 34.767, Francia con 29.843, España con 28.355, y México con 27.121. 
Tabla 3. Comparaciones múltiples. Prueba de Duncan. Subconjuntos 1 al 11

\begin{tabular}{|l|l|l|l|l|l|l|l|l|l|l|l|l|l|l|}
\hline \multicolumn{1}{|c|}{ País } & N & 1 & 2 & 3 & 4 & 5 & 6 & 7 & 8 & 9 & 10 & 11 \\
\hline Suiza & 3 & 31628 & & & & & & & & & & \\
Polonia & 3 & 33986 & 33986 & & & & & & & & & \\
Filipinas & 3 & 36110 & 36110 & 36110 & & & & & & & & \\
Omán & 3 & 39093 & 39093 & 39093 & & & & & & & & \\
Portugal & 3 & 41582 & 41582 & 41582 & 41582 & & & & & & & \\
Ucrania & 3 & 43197 & 43197 & 43197 & 43197 & & & & & & & \\
Singapur & 3 & 43675 & 43675 & 43675 & 43675 & & & & & & & \\
Kuwait & 3 & 45553 & 45553 & 45553 & 45553 & & & & & & & \\
Irak & 3 & & 47220 & 47220 & 47220 & 47220 & & & & & & \\
Emiratos Árabes & 3 & & 48096 & 48096 & 48096 & 48096 & 48096 & & & & & \\
Holanda & 3 & & & 50172 & 50172 & 50172 & 50172 & & & & & \\
Ecuador & 3 & & & & 55071 & 55071 & 55071 & 55071 & & & & \\
Indonesia & 3 & & & & 55162 & 55162 & 55162 & 55162 & & & & \\
Argentina & 3 & & & & & 59968 & 59968 & 59968 & & & & \\
Bélgica & 3 & & & & & 61317 & 61317 & 61317 & & & & \\
Bielorrusia & 3 & & & & & & 61794 & 61794 & & & & \\
Egipto & 3 & & & & & & & 65710 & & & & \\
Suecia & 3 & & & & & & & 67085 & & & & \\
China & 3 & & & & & & & & 83514 & & & \\
Colombia & 3 & & & & & & & & 94886 & 94886 & & \\
Qatar & 3 & & & & & & & & 95202 & 95202 & & \\
Canadá & & & & & & & & & & 103537 & & \\
Bangladesh & & & & & & & & & & & 140352 & \\
Sudáfrica & & & & & & & & & & & 144535 & \\
Francia & & & & & & & & & & & & 163999 \\
Sig. & & 0,056 & 0,055 & 0,056 & 0,065 & 0,050 & 0,057 & 0,097 & 0,07 & 0,191 & 0,500 & 1,000 \\
\hline
\end{tabular}

Se visualizan las medias para los grupos en los subconjuntos homogéneos. Utiliza el tamaño de la muestra de la media armónica $=3,000$. Fuente: el autor.

Tabla 4. Comparaciones múltiples. Prueba de Duncan. Subconjuntos 12 al 22

\begin{tabular}{|c|c|c|c|c|c|c|c|c|c|c|c|c|}
\hline País & N & 12 & 13 & 14 & 15 & 16 & 17 & 18 & 19 & 20 & 21 & 22 \\
\hline Arabia Saudita & 3 & 186603 & & & & & & & & & & \\
\hline Alemania & 3 & 195317 & 195317 & & & & & & & & & \\
\hline Turquía & 3 & 198586 & 198586 & & & & & & & & & \\
\hline Pakistán & 3 & & 206267 & 206267 & & & & & & & & \\
\hline México & 3 & & & 216770 & 216770 & & & & & & & \\
\hline Irán & 3 & & & & 225178 & & & & & & & \\
\hline Italia & 3 & & & & & 240441 & & & & & & \\
\hline España & 3 & & & & & 249003 & & & & & & \\
\hline Chile & 3 & & & & & & 275791 & & & & & \\
\hline Perú & 3 & & & & & & 279257 & & & & & \\
\hline Reino Unido & 3 & & & & & & & 311923 & & & & \\
\hline India & 3 & & & & & & & & 548005 & & & \\
\hline Rusia & 3 & & & & & & & & & 641147 & & \\
\hline Brasil & 3 & & & & & & & & & & 1353295 & \\
\hline Estados Unidos & 3 & & & & & & & & & & & 2628930 \\
\hline Sig. & & 0,070 & 0,098 & 0,093 & 0,178 & 0,170 & 0,576 & 1,000 & 1,000 & 1,000 & 1,000 & 1,000 \\
\hline
\end{tabular}

Se visualizan las medias para los grupos en los subconjuntos homogéneos. a. Utiliza el tamaño de la muestra de la media armónica = 3,000. Fuente: el autor. 


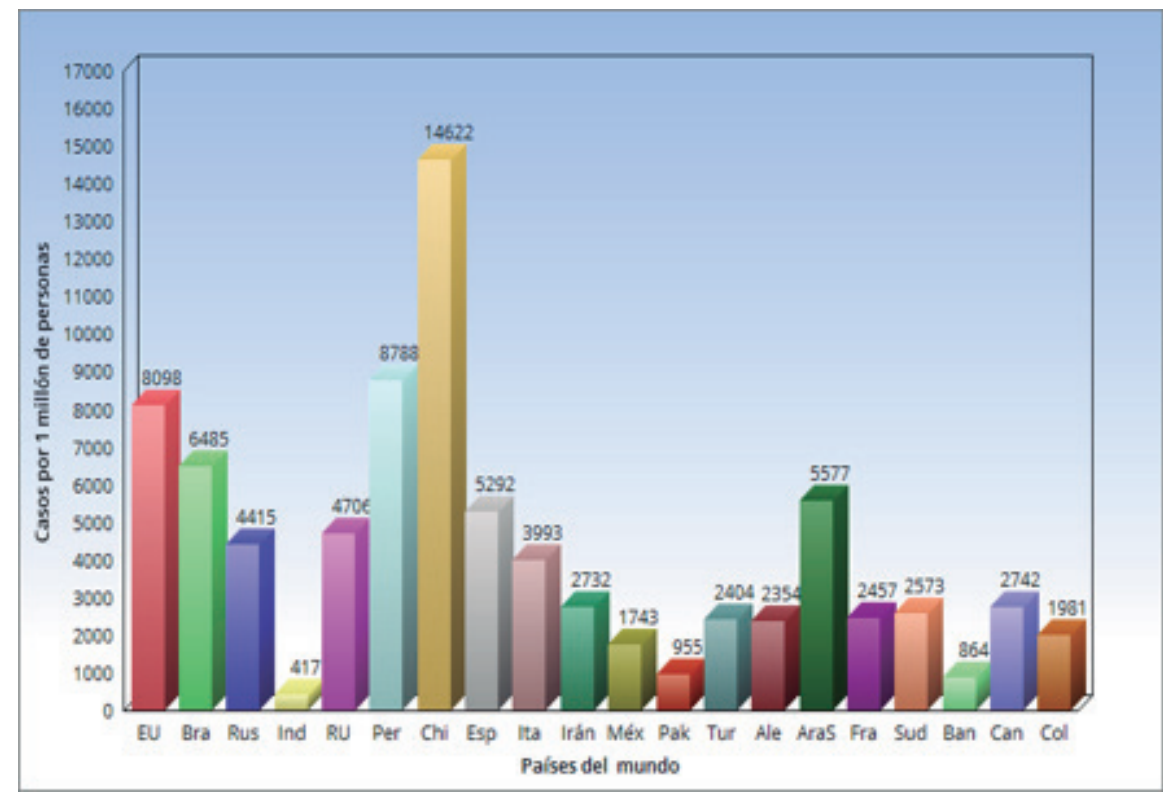

Figura 1. Casos de contagio por COVID-19, por 1 millón de personas. Fuente: el autor.

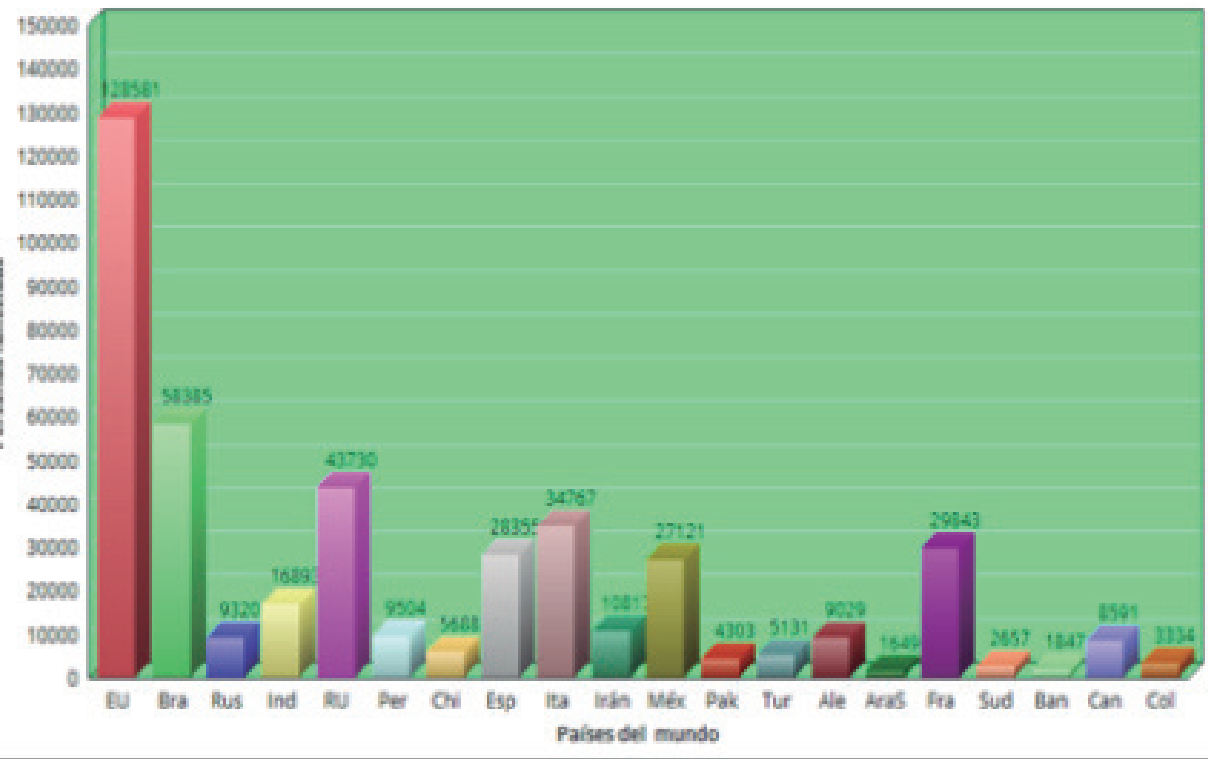

Figura 2. Personas fallecidas por COVID-19 a nivel mundial. Fuente: el autor.

\section{CONCLUSIONES}

A partir del análisis estadístico de los datos aplicando la comparación de medias con el ANOVA, el p-valor de las pruebas, para la variable contagios es de 0,000, este es menor a $a=0.05$, de esta manera se rechaza Ho, es decir que para al menos uno de los promedios es diferente de los demás del resultado obtenido para los diferentes países del mundo por contagio de personas por COVID-19, entre el periodo comprendido entre el 28 y 30 de junio de 2020.

Se evidenció que los países con menor promedio de personas contagiadas son: Suiza 31.628, Polonia 33.986, Filipinas 36.110, Omán 39.093, Portugal 41.582, Ucrania 43.197, Singapur 43.675, Kuwait 45.553. Los países con mayor promedio de personas contagiadas por el COVID-19 son: Estados Unidos 2'628.930, seguida de Brasil 1'353.295, 
Rusia 641.147, India 584.005, y Reino Unido 311.923. Ahora para el mayor número de casos por 1 millón de personas a 30 de junio 2020, Chile obtuvo el mayor índice 14.622, Perú 8.788, Estados Unidos 8.098, Brasil 6.485, Arabia Saudita 5.577, y España 5.292.

Para el mayor número de personas fallecidas a 30 de junio 2020, lideró los Estados Unidos 128.581, Brasil 58.385, Rusia 47.730, Italia 34.767, Francia 29.843, España 28.355, y México 27.121. El nuevo coronavirus (COVID-19) está retando al mundo. Sin una vacuna y una capacidad médica limitada para tratar la enfermedad, las intervenciones no farmacéuticas (NPI) son la estrategia principal para inmovilizar la pandemia. Las restricciones globales de viaje sin precedentes y las órdenes de quedarse en casa están originando la interrupción más grave de la economía global desde la Segunda Guerra Mundial. ${ }^{14}$

\section{REFEREN CIAS}

1. Addo PC, Jiaming F, Kulbo NB, Liangqiang L. COVID-19: fear appeal favoring purchase behavior towards personal protective equipment. The Service Industries Journal. 2020;40(7-8):471-90. doi: https://doi.org/10.1080/02642069.2020.1751823

2. Fang B, Meng QH. The laboratory's role in combating COVID-19. Critical Reviews in Clinical Laboratory Sciences. 2020:1-15. doi: https://doi.org/10.1080/10408363.2020.1776675

3. Desouky E. Urological surgery in the COVID-19 era: Patient counselling and informed consent. Arab Journal of Urology. 2020;18(2):62-4. doi: https://doi.org/10.1080/2090598X.2020.1772032

4. Dryhurst S, Schneider CR, Kerr J, Freeman ALJ, Recchia G, van der Bles AM, et al. Risk perceptions of COVID-19 around the world. Journal of Risk Research. 2020:1-13. doi: https://doi.org/10.1080/ 13669877.2020.1758193
5. Sharfuddin S. The world after Covid-19. The Round Table. 2020;109(3):247-57. doi: https://doi.org/10.1080/00358533.2020. 1760498

6. Díaz-Pinzón JE. Estudio comparativo entre el contagio durante la cuarentena obligada por el COVID-19 y el contagio durante la apertura gradual y controlada para algunos sectores de la economía en Colombia. Repert Med Cir. 2020;29(Supl. Núm. 1):52-8. doi: : https://doi.org/10.31260/RepertMedCir.01217372.1073

7. Chung CKL, Xu J, Zhang M. Geographies of Covid-19: how space and virus shape each other. Asian Geographer. 2020:1-18. doi: https://doi.org/10.1080/10225706.2020.1767423

9. Pinzón, JE. Aplicación Phet: estrategia de enseñanza aprendizaje de fracciones equivalentes. Revista Criterios. 2016;23(1):99-111.

10. Google. Coronavirus (COVID-19) [Internet]. 2020 [citado 24 de junio de 2020]; Disponible en: https://news.google.com/covid19/ map?hl=es419\&gl=CO\&ceid=CO:es-419

11. Llopis, Jaume. Test de Duncan [Internet]. [citado 24 de junio de 2020]; Disponible en: https://jllopisperez.com/2013/01/28/test-deduncan/

12. Instituto Nacional de Salud de Colombia. Coronavirus (COVID-19) en Colombia [Internet]. 2020 [Citado 04 de julio de 2020]. Disponible en: https://bit.ly/2UNnOtl

13. Organización Mundial de la Salud. Brote de enfermedad por Coronavirus. (COVID-19) [Internet]. 2020 [Citado 04 de julio de 2020]. Disponible en: https://www.who.int/es/emergencies/ diseases/novel-coronavirus-2019? gclid=EAIaIQobChMIt_ bMxt6z6gIViI7ICh3hZw4NEAAYASAAEgIhsPD_BwE

14. Gössling S, Scott D, Hall CM. Pandemics, tourism and global change: a rapid assessment of COVID-19. Journal of Sustainable Tourism. 2020:1-20. doi: https://doi.org/10.1080/09669582.2020. 1758708 